\title{
The twisted Floer homology of torus bundles
}

\author{
YINGHUA AI \\ THOMAS D PETERS
}

\begin{abstract}
We prove an exact sequence for $\omega$-twisted Heegaard Floer homology. As a corollary, given a torus bundle $Y$ over the circle and a cohomology class $[\omega] \in H^{2}(Y ; \mathbb{Z})$ which evaluates nontrivially on the fiber, we compute the Heegaard Floer homology of $Y$ with twisted coefficients in the universal Novikov ring.
\end{abstract}

57M27; 53D40

\section{Introduction}

Heegaard Floer homology was introduced by Ozsváth and Szabó in [17; 16]. It provides powerful invariants for closed oriented 3-manifolds. These invariants come as a package of abelian groups denoted $H F^{\circ}$. There is also a filtered version, called knot Floer homology, for null-homologous knots defined by Ozsváth and Szabó [15] as well as Rasmussen [22]. The Heegaard Floer homology groups reflect many interesting geometric properties of the 3-manifold. For instance, Ozsváth and Szabó showed that they detect the Thurston seminorm on a closed oriented 3-manifold [14, Theorem 1.1]. As another example, work of Ghiggini [4] and Ni [11] shows that knot Floer homology detects fiberedness in knots.

Turning to closed fibered 3-manifolds, note that a 3-manifold which admits a fibration $\pi: Y \rightarrow S^{1}$ has a canonical $\operatorname{Spin}^{c}$ structure, $\ell$, obtained as the tangents to the fibers of $\pi$.

Theorem 1.1 (Ozsváth-Szabó [18]) Let $Y$ be a closed 3-manifold which fibers over the circle, with fiber $F$ of genus $g>1$, and let $\mathfrak{t}$ be a $\operatorname{Spin}^{c}$ structure over $Y$ with

$$
\left\langle c_{1}(\mathfrak{t}),[F]\right\rangle=2-2 g .
$$

Then for $\mathfrak{t} \neq \ell$, we have that

while

$$
\begin{aligned}
& H F^{+}(Y, \mathfrak{t})=0 \\
& H F^{+}(Y, \ell) \cong \mathbb{Z} .
\end{aligned}
$$


This is commonly refereed to as the fact that the Floer homology of a surface bundle (with fiber genus greater than one) is "monic" in its "top-most" Spin ${ }^{c}$-structure. In fact, Yi Ni proved a converse to Theorem 1.1:

Theorem 1.2 (Ni [12]) Suppose $Y$ is a closed irreducible 3-manifold, $F \subset Y$ is a closed connected surface of genus $g>1$. Let $\mathrm{HF}^{+}(Y,[F], 1-g)$ denote the group

$$
\bigoplus_{\substack{\mathfrak{s} \in \operatorname{Spin}^{c}(Y) \\\left\langle c_{1}(\mathfrak{s}),[F]\right\rangle=2-2 g}} H F^{+}(Y, \mathfrak{s}) .
$$

If $\mathrm{HF}^{+}(Y,[F], 1-g) \cong \mathbb{Z}$, then $Y$ fibers over the circle with $F$ as a fiber.

For a fiber bundle with torus fiber $F, H^{+}(Y,[F], 0)$ is always infinitely generated as an abelian group. However, as we shall show, if one works with Floer homology and an appropriate version of Novikov coefficients, the Floer homology of a torus bundle is still "monic" in a certain sense. Much is already known about the Floer homology of torus bundles. For instance, John Baldwin has already computed the untwisted Heegaard Floer homologies of torus bundles with $b_{1}(Y)=1$ in [2].

In this paper, we use Heegaard Floer homology with twisted coefficients in the universal Novikov ring, $\Lambda$, of all formal power series with real coefficients of the form

$$
f(t)=\sum_{r \in \mathbb{R}} a_{r} t^{r} \quad \text { such that } \#\left\{r \in \mathbb{R} \mid a_{r} \neq 0, r \leq c\right\}<\infty \text { for all } c \in \mathbb{R} .
$$

Given a cohomology class $[\omega] \in H^{2}(Y ; \mathbb{Z}), \Lambda$ can be given a $\mathbb{Z}\left[H^{1}(Y ; \mathbb{Z})\right]$-module structure, and this gives rise to a twisted Heegaard Floer homology $\underline{H F}^{+}\left(Y ; \Lambda_{\omega}\right)$. This version was first defined by Ozsváth and Szabó in [17, Section 10] and can also be derived from the definition of general twisted Heegaard Floer homology in [16]. We will describe this group explicitly in Section 2.1. It is worth noting that Heegaard Floer homology with twisted coefficients in a certain Novikov ring has already been studied extensively by Jabuka and Mark [7]. The main theorem we prove in this paper is the following:

Theorem 1.3 Suppose $Y$ is a closed oriented 3-manifold which fibers over the circle with torus fiber $F$, and $[\omega] \in H^{2}(Y ; \mathbb{Z})$ is a cohomology class such that $\omega(F) \neq 0$. Then we have an isomorphism of $\Lambda$-modules

$$
\underline{H F}^{+}\left(Y ; \Lambda_{\omega}\right) \cong \Lambda \text {. }
$$

Remark 1.4 In the setting of Monopole Floer homology, a corresponding version of this theorem was proved by Kronheimer and Mrowka in [8, Theorem 42.7.1]. 
Theorem 1.3 was also proved using different methods by Lekili [10, Theorem 12]. In fact, he actually proved the stronger statement that for a torus bundle $Y, \underline{H F}^{+}\left(Y ; \Lambda_{\omega}\right)$ is supported in the canonical $\operatorname{Spin}^{c}$-structure $\ell$. Also, Ai and Ni proved in [1] that the converse of the above theorem also holds, ie the twisted Heegaard Floer homology determines whether an irreducible 3-manifold is a torus bundle over the circle.

This paper is organized as follows. We provide a review of Heegaard Floer homology with twisted coefficients in Section 2, including the most pertinent example, $S^{1} \times S^{2}$. In Section 3 we prove a relevant exact triangle for $\omega$-twisted Heegaard Floer homology and prove Theorem 1.3.

Acknowledgements The authors would like to thank Peter Ozsváth for his continued guidance and support. The first author would also like to thank Yi Ni for suggesting the problem and providing some key ideas.

This work was carried out while the first author was an exchange graduate student at Columbia University, supported by the China Scholarship Council. He is grateful to the Columbia University Mathematics Department for its hospitality.

Finally, we would like to thank the anonymous referee for a careful reading of our draft and for many helpful comments and suggestions.

That said, any remaining errors are solely the responsibility of the authors.

\section{Review of twisted coefficients}

We recall the construction of Heegaard Floer homology with twisted coefficients; see Ozsváth and Szabó $[16 ; 14]$ for more details. To a closed oriented 3 -manifold $Y$ we associate a pointed Heegaard diagram $(\Sigma, \boldsymbol{\alpha}, \boldsymbol{\beta}, z)$, where $\Sigma$ is an oriented surface of genus $g \geq 1$ and $\boldsymbol{\alpha}=\left\{\alpha_{1}, \ldots, \alpha_{g}\right\}$ and $\boldsymbol{\beta}=\left\{\beta_{1}, \ldots, \beta_{g}\right\}$ are sets of attaching circles (assumed to intersect transversely) for the two handlebodies in the Heegaard decomposition. These give a pair of transversely intersecting $g$-dimensional tori $\mathbb{T}_{\alpha}=\alpha_{1} \times \cdots \times \alpha_{g}$ and $\mathbb{T}_{\beta}=\beta_{1} \times \cdots \times \beta_{g}$ in the symmetric product $\operatorname{Sym}^{g}(\Sigma)$. Recall that the basepoint $z$ gives a map $\mathfrak{s}_{z}: \mathbb{T}_{\alpha} \cap \mathbb{T}_{\beta} \rightarrow \operatorname{Spin}^{c}(Y)$. Given a $\operatorname{Spin}^{c}$ structure $\mathfrak{s}$ on $Y$, let $\mathfrak{S} \subset \mathbb{T}_{\alpha} \cap \mathbb{T}_{\beta}$ be the set of intersection points $\mathbf{x} \in \mathbb{T}_{\alpha} \cap \mathbb{T}_{\beta}$ such that $\mathfrak{s}_{z}(\mathbf{x})=\mathfrak{s}$.

Given intersection points $\mathbf{x}$ and $\mathbf{y}$ in $\mathbb{T}_{\alpha} \cap \mathbb{T}_{\beta}$, let $\pi_{2}(\mathbf{x}, \mathbf{y})$ denote the set of homotopy classes of Whitney disks from $\mathbf{x}$ to $\mathbf{y}$. There is always a natural map from $\pi_{2}(\mathbf{x}, \mathbf{x})$ to $H^{1}(Y ; \mathbb{Z})$ obtained as follows: each $\phi \in \pi_{2}(\mathbf{x}, \mathbf{x})$ naturally gives rise to an associated two-chain in $\Sigma$ whose boundary is a collection of circles among the $\boldsymbol{\alpha}$ and $\boldsymbol{\beta}$-curves. 
We then close off this two-chain by gluing copies of the attaching disks for the handlebodies in the Heegaard diagram for $Y$. The Poincaré dual of this two-cycle is the associated element of $H^{1}(Y ; \mathbb{Z})$.

Given a $\operatorname{Spin}^{c}$ structure $\mathfrak{s}$ on $Y$ and a Heegaard diagram $(\Sigma, \boldsymbol{\alpha}, \boldsymbol{\beta}, z)$ for $Y$, an additive assignment is a collection of maps

$$
A=\left\{A_{\mathbf{x}, \mathbf{y}}: \pi_{2}(\mathbf{x}, \mathbf{y}) \rightarrow H^{1}(Y ; \mathbb{Z})\right\}_{\mathbf{x}, \mathbf{y} \in \mathfrak{S}}
$$

with the following properties:

- When $\mathbf{x}=\mathbf{y}, A_{\mathbf{x}, \mathbf{x}}$ is the canonical map from $\pi_{2}(\mathbf{x}, \mathbf{x})$ onto $H^{1}(Y ; \mathbb{Z})$ defined above.

- A is compatible with splicing in the sense that if $\mathbf{x}, \mathbf{y}, \mathbf{u} \in \mathfrak{S}$ then for each $\phi_{1} \in \pi_{2}(\mathbf{x}, \mathbf{y})$ and $\phi_{2} \in \pi_{2}(\mathbf{y}, \mathbf{u})$, we have that $A\left(\phi_{1} * \phi_{2}\right)=A\left(\phi_{1}\right)+A\left(\phi_{2}\right)$.

- $A_{\mathbf{x}, \mathbf{y}}(S * \phi)=A_{\mathbf{x}, \mathbf{y}}(\phi)$ for $S \in \pi_{2}\left(\operatorname{Sym}^{g}\left(\Sigma_{g}\right)\right)$.

Additive assignments may be constructed with the help of a complete system of paths as described in Ozsváth-Szabó [16].

We write elements in the group-ring $\mathbb{Z}\left[H^{1}(Y ; \mathbb{Z})\right]$ as finite formal sums

$$
\sum_{g \in H^{1}(Y ; \mathbb{Z})} n_{g} \cdot e^{g}
$$

for $n_{g} \in \mathbb{Z}$. The universally twisted Heegaard Floer complex

$$
\underline{C F}^{\infty}\left(Y, \mathfrak{s} ; \mathbb{Z}\left[H^{1}(Y ; \mathbb{Z})\right], A\right)
$$

is the free $\mathbb{Z}\left[H^{1}(Y ; \mathbb{Z})\right]$-module on generators $[\mathbf{x}, i]$ for $\mathbf{x} \in \mathfrak{S}$ and $i \in \mathbb{Z}$. The differential, $\underline{\partial}^{\infty}$, is given by

$$
\underline{\partial}^{\infty}[x, i]=\sum_{\mathbf{y} \in \mathfrak{S}} \sum_{\substack{\phi \in \pi_{2}(\mathbf{x}, \mathbf{y}) \\ \mu(\phi)=1}} \# \widehat{\mathcal{M}}(\phi) \cdot e^{A(\phi)} \otimes\left[\mathbf{y}, i-n_{z}(\phi)\right] .
$$

Here $\mu(\phi)$ denotes the Maslov index of $\phi$, the formal dimension of the space $\mathcal{M}(\phi)$ of pseudo-holomorphic representatives in the homotopy class of $\phi, n_{z}(\phi)$ denotes the intersection number of $\phi$ with the subvariety $\{z\} \times \operatorname{Sym}^{g-1}(\Sigma) \subset \operatorname{Sym}^{g}(\Sigma)$, and $\widehat{\mathcal{M}}(\phi)$ denotes the quotient of $\mathcal{M}(\phi)$ under the natural action of $\mathbb{R}$. To ensure that the sums appearing in the definition of the differential are finite, one must restrict to $\mathfrak{s}$-admissible Heegaard diagrams, as defined in Ozsváth-Szabó [17, Section 4.2.2]. Just as in the untwisted setting, this complex admits a $\mathbb{Z}[U]$-action via $U:[\mathbf{x}, i] \mapsto[\mathbf{x}, i-1]$. This gives rise to variants $\underline{C F^{+}}, \underline{C F^{-}}$, and $\underline{\widehat{C F}}$, denoted collectively as $\underline{C F^{\circ}}$. The homology 
groups of these complexes are the universally twisted Heegaard Floer homology groups $\underline{H F^{\circ}}$.

More generally, given any $\mathbb{Z}\left[H^{1}(Y ; \mathbb{Z})\right]$-module $M$, we may form Floer homology groups with coefficients in $M$ by taking $\underline{H F^{\circ}}(Y, \mathfrak{s} ; M)$ as the homology of the complex

$$
\underline{C F^{\circ}}(Y, \mathfrak{s} ; M, A):=\underline{C F^{\circ}}\left(Y, \mathfrak{s} ; \mathbb{Z}\left[H^{1}(Y ; \mathbb{Z})\right], A\right) \otimes_{\mathbb{Z}\left[H^{1}(Y ; \mathbb{Z})\right]} M .
$$

For instance, by taking $M=\mathbb{Z}$, thought of as being a trivial $\mathbb{Z}\left[H^{1}(Y ; \mathbb{Z})\right]$-module, one recovers the ordinary untwisted Heegaard Floer homology, $C F^{\circ}(Y, \mathfrak{s})$.

In Ozsváth-Szabó [17], it is proved that the homologies defined above are independent of the choice of additive assignment $A$ and are topological invariants of the pair $(Y, \mathfrak{s})$. As in the untwisted setting, these groups are related by long exact sequences:

$$
\begin{gathered}
\cdots \longrightarrow \underline{\widehat{H F}}(Y, \mathfrak{s} ; M) \longrightarrow \underline{H F^{+}}(Y, \mathfrak{s} ; M) \stackrel{U}{\longrightarrow} \underline{H F^{+}}(Y, \mathfrak{s} ; M) \longrightarrow \cdots \\
\cdots \longrightarrow \underline{H F}(Y, \mathfrak{s} ; M) \longrightarrow \stackrel{\iota}{\longrightarrow} \underline{H F}{ }^{\infty}(Y, \mathfrak{s} ; M) \stackrel{\pi}{\longrightarrow} \underline{H F^{+}}(Y, \mathfrak{s} ; M) \longrightarrow \cdots
\end{gathered}
$$

Of course, the chain complex $C F^{\circ}(Y, \mathfrak{s} ; M)$ is obtained from the chain complex in the universally twisted case, $C F^{\circ}\left(Y, \mathfrak{s} ; \mathbb{Z}\left[H^{1}(Y ; \mathbb{Z})\right]\right)$, by a change of coefficients and hence the corresponding homology groups are related by the universal coefficients spectral sequence (see for instance Cartan and Eilenberg [3]).

\section{$2.1 \omega$-Twisted Heegaard Floer homology}

In this section we briefly recall the notion of $\omega$-twisted Heegaard Floer homology, following Ozsváth-Szabó $[17 ; 14]$.

The universal Novikov ring is defined to be the set of all formal power series with real coefficients of the form

$$
f(t)=\sum_{r \in \mathbb{R}} a_{r} t^{r} \quad \text { such that } \#\left\{r \in \mathbb{R} \mid a_{r} \neq 0, r \leq c\right\}<\infty \text { for all } c \in \mathbb{R} .
$$

It is endowed with the following multiplication, making it into a field:

$$
\left(\sum_{r \in \mathbb{R}} a_{r} t^{r}\right) \cdot\left(\sum_{r \in \mathbb{R}} b_{r} t^{r}\right)=\sum_{r \in \mathbb{R}}\left(\sum_{s \in \mathbb{R}} a_{s} b_{r-s}\right) t^{r} .
$$

Furthermore, by fixing a cohomology class $[\omega] \in H^{2}(Y ; \mathbb{R})$ we can give $\Lambda$ a $\mathbb{Z}\left[H^{1}(Y ; \mathbb{Z})\right]$-module structure via the ring homomorphism

$$
\begin{aligned}
\mathbb{Z}\left[H^{1}(Y ; \mathbb{Z})\right] & \rightarrow \Lambda \\
\sum a_{h} \cdot e^{h} & \mapsto \sum a_{h} \cdot t^{\langle h \cup \omega,[Y]\rangle} .
\end{aligned}
$$


When we are interested in its $\mathbb{Z}\left[H^{1}(Y ; \mathbb{Z})\right]$-module structure, we denote $\Lambda$ by $\Lambda_{\omega}$. This $\mathbb{Z}\left[H^{1}(Y ; \mathbb{Z})\right]$-module structure gives rise to a twisted Heegaard Floer homology $\underline{H F}^{+}\left(Y ; \Lambda_{\omega}\right)$, which we refer to as $\omega$-twisted Heegaard Floer homology. More concretely, it can be defined as follows (see Ozsváth-Szabó [14, Section 3.1]). Choose a weakly admissible pointed Heegaard diagram $(\Sigma, \boldsymbol{\alpha}, \boldsymbol{\beta}, z)$ for $Y$ and fix a 2-cocycle representative $\omega \in[\omega]$. Every Whitney disk $\phi$ in $\operatorname{Sym}^{g}(\Sigma)$ (for $\mathbb{T}_{\alpha}$ and $\mathbb{T}_{\beta}$ ) gives rise to a two-chain $[\phi]$ in $Y$ by coning off partial $\alpha$ and $\beta$-circles with gradient trajectories in the $\alpha$ and $\beta$ handlebodies. The evaluation of $\omega$ on $[\phi]$ depends only on the homotopy class of $\phi$ and is denoted $\int_{[\phi]} \omega$ (or sometimes $\omega([\phi])$ ). The $\omega$-twisted chain complex $\underline{C F^{+}}\left(Y ; \Lambda_{\omega}\right)$ is the free $\Lambda$-module generated by $[x, i]$ with $x \in \mathbb{T}_{\alpha} \cap \mathbb{T}_{\beta}$ and integers $i \geq 0$, endowed with the following differential:

$$
\underline{\partial}^{+}[\mathbf{x}, i]=\sum_{\mathbf{y} \in \mathbb{T}_{\alpha} \cap \mathbb{T}_{\beta}} \sum_{\left\{\phi \in \pi_{2}(\mathbf{x}, \mathbf{y}) \mid \mu(\phi)=1\right\}} \# \widehat{\mathcal{M}}(\phi)\left[\mathbf{y}, i-n_{z}(\phi)\right] \cdot t_{[\phi]}^{\int_{[\phi} \omega}
$$

Its homology is the $\omega$-twisted Heegaard Floer homology $\underline{H F}^{+}\left(Y ; \Lambda_{\omega}\right)$. Notice that this group is both a module for $\mathbb{Z}\left[H^{1}(Y ; \mathbb{Z})\right]$ and a module for $\Lambda$. Notice also that although the differential depends on the choice of 2-cocycle representative $\omega \in[\omega]$, the isomorphism class of the chain complex only depends on the cohomology class. This may be seen as follows: suppose we have cohomologous 2 -forms $\omega_{1}$ and $\omega_{2}$ on $Y$. Fixing an intersection point $\mathbf{x}_{0} \in \mathbb{T}_{\alpha} \cap \mathbb{T}_{\beta}$, define $\Phi: \underline{C F^{\infty}}\left(Y ; \Lambda_{\omega_{1}}\right) \rightarrow \underline{C F}^{\infty}\left(Y ; \Lambda_{\omega_{2}}\right)$ by $\Phi([\mathbf{x}, i])=[\mathbf{x}, i] t^{\left(\omega_{2}-\omega_{1}\right)\left[\phi_{x}\right]}$ where $\phi_{x}$ is any element in $\pi_{2}\left(\mathbf{x}_{0}, \mathbf{x}\right)$ (the choice is irrelevant: the associated domains of any two choices would differ by a periodic domain, which then caps off to a closed surface on which the exact form $\omega_{2}-\omega_{1}$ evaluates to zero). It is then an easy exercise to see that $\Phi$ induces a chain isomorphism. An advantage of using this viewpoint is that we avoid altogether the notion of an "additive assignment". It is easy to see (using an argument similar to the previous), that the complex defined above is isomorphic to one obtained by choosing an additive assignment and then tensoring with the $\mathbb{Z}\left[H^{1}(Y ; \mathbb{Z})\right]$-module $\Lambda_{\omega}$.

Suppose $W: Y_{1} \rightarrow Y_{2}$ is a 4-dimensional cobordism from $Y_{1}$ to $Y_{2}$ given by a single $2-$ handle addition and we have a cohomology class $[\omega] \in H^{2}(W ; \mathbb{R})$. Then there is an associated Heegaard triple $(\Sigma, \boldsymbol{\alpha}, \boldsymbol{\beta}, \boldsymbol{\gamma}, z)$ and 4 -manifold $X_{\alpha \beta \gamma}$ representing $W$ minus a one-complex (see Ozsváth-Szabó [20, Proposition 4.3]). Similar to before, a Whitney triangle $\psi \in \pi_{2}(\mathbf{x}, \mathbf{y}, \mathbf{w})$ determines a two-chain in $X_{\alpha \beta \gamma}$ on which we may evaluate a representative, $\omega \in[\omega]$. As before, this evaluation depends only on the homotopy class of $\psi$ and is denoted by $\int_{[\psi]} \omega$. This gives rise to a $\Lambda$-equivariant map

$$
\underline{F}_{W ; \omega}^{+}: \underline{H F}^{+}\left(Y_{1} ; \Lambda_{\left.\omega\right|_{Y_{1}}}\right) \rightarrow \underline{H F}^{+}\left(Y_{2} ; \Lambda_{\left.\omega\right|_{Y_{2}}}\right)
$$


(which is defined only up to multiplication by $\pm t^{c}$ for some $c \in \mathbb{R}$ ) defined on the chain level by

$$
\underline{f}_{W ; \omega}^{+}[\mathbf{x}, i]=\sum_{\mathbf{y} \in \mathbb{T}_{\alpha} \cap \mathbb{T}_{\gamma}} \sum_{\substack{\psi \in \pi_{2}(\mathbf{x}, \Theta, \mathbf{y}) \\ \mu(\psi)=0}} \# \mathcal{M}(\psi)\left[\mathbf{y}, i-n_{z}(\psi)\right] \cdot t^{\int_{[\psi]}} \omega
$$

where $\Theta \in \mathbb{T}_{\beta} \cap \mathbb{T}_{\gamma}$ represents a top dimensional generator for the Floer homology $H F^{\leq 0}\left(Y_{\beta \gamma}\right) \cong \wedge^{*} H^{1}\left(Y_{\beta \gamma}\right) \otimes \mathbb{Z}[U]$ of the 3 -manifold determined by the Heegaard diagram $(\Sigma, \boldsymbol{\beta}, \boldsymbol{\gamma}, z)$, which is a connected sum $\#^{g-1}\left(S^{1} \times S^{2}\right)(g$ denotes the genus of $\Sigma$ ), and $\mathcal{M}(\psi)$ denotes the moduli space of pseudo-holomorphic triangles in the homotopy class $\psi$. This definition may be extended to arbitrary smooth, connected cobordisms as in Ozsváth-Szabó [20]. These maps may be decomposed as a sum of maps

$$
\underline{F}_{W ; \omega}^{+}=\sum_{\mathfrak{s} \in \operatorname{Spin}^{c}(W)} \underline{F}_{W, \mathfrak{s} ; \omega}^{+}
$$

which are summed according to $\operatorname{Spin}^{c}$ equivalence classes of triangles, just as in the untwisted setting. This can be extended to arbitrary (smooth, connected) cobordisms from $Y_{1}$ to $Y_{2}$ as in Ozsváth-Szabó [20]. These maps also satisfy a composition law: if $W_{1}$ is a cobordism from $Y_{1}$ to $Y_{2}$ and $W_{2}$ is a cobordism from $Y_{2}$ to $Y_{3}$, and we equip $W_{1}$ and $W_{2}$ with $\operatorname{Spin}^{c}$ structures $\mathfrak{s}_{1}$ and $\mathfrak{s}_{2}$ respectively (whose restrictions agree over $\left.Y_{2}\right)$, then putting $W=W_{1} \#_{Y_{2}} W_{2}$, for any $[\omega] \in H^{2}(W ; \mathbb{R})$ there are choices of representatives for the cobordism maps such that:

$$
\underline{F}_{W_{2}, \mathfrak{s}_{2} ;\left.\omega\right|_{W_{2}}}^{+} \circ \underline{F}_{W_{1}, \mathfrak{s}_{1} ;\left.\omega\right|_{W_{1}}}^{+}=\sum_{\left\{\mathfrak{s} \in \operatorname{Spin}^{c}(W):\left.\mathfrak{s}\right|_{W_{i}}=\mathfrak{s}_{i}\right\}} \underline{F}_{W, \mathfrak{s} ; \omega}^{+}
$$

\subsection{Example: $S^{1} \times S^{2}$}

In this section we calculate twisted Heegaard Floer homologies of $S^{1} \times S^{2}$. We start with the universally twisted version $\underline{\widehat{H F}}\left(S^{1} \times S^{2} ; \mathbb{Z}\left[t, t^{-1}\right]\right)$, where we have identified $\mathbb{Z}\left[H^{1}\left(S^{1} \times S^{2} ; \mathbb{Z}\right)\right] \cong \mathbb{Z}\left[t, t^{-1}\right]$, the ring of Laurent polynomials. $S^{1} \times S^{2}$ has a standard genus-one Heegaard decomposition $(\Sigma, \alpha, \beta)$ where $\alpha$ is a homotopically nontrivial embedded curve and $\beta$ is an isotopic translate. For simplicity, we only compute $\underline{\widehat{H F}}$. We make the diagram weakly admissible for the unique torsion $\operatorname{Spin}^{c}$ structure $\mathfrak{s}_{0}$ by introducing cancelling pairs of intersection points between $\alpha$ and $\beta$. This gives a pair of intersection points $x^{+}$and $x^{-}$. We next need an additive assignment. Notice there is an obvious periodic domain consisting of a pair of (nonhomotopic) disks $D_{1}$ and $D_{2}$ connecting $x^{+}$and $x^{-}$. When capped off, the periodic domain gives a sphere representing a generator of $H_{2}\left(S^{1} \times S^{2} ; \mathbb{Z}\right) \cong \mathbb{Z}$. Hence taking Poincaré dual we recover a generator of $H^{1}\left(S^{1} \times S^{2} ; \mathbb{Z}\right) \cong \mathbb{Z}$. We place the basepoint $z$ in the 
complement of the disks $D_{1}$ and $D_{2}$. Identifying $H^{1}\left(S^{1} \times S^{2} ; \mathbb{Z}\right) \cong \mathbb{Z}$, an additive assignment must assign 1 to this domain. One way this can be done is by assigning 1 to $D_{1}$ and 0 to $D_{2}$. Applying the Riemann mapping theorem, and the argument given on page 1169 of Ozsváth-Szabó [16], we see that the complex $\widehat{C F}\left(S^{1} \times S^{2} ; \mathbb{Z}\left[t, t^{-1}\right]\right)$ is just:

$$
0 \longrightarrow \mathbb{Z}\left[t, t^{-1}\right] \stackrel{1-t}{\longrightarrow} \mathbb{Z}\left[t, t^{-1}\right] \longrightarrow 0
$$

Here, the first copy of $\mathbb{Z}\left[t, t^{-1}\right]$ corresponds to $x^{+}$and the second corresponds to $x^{-}$. This complex has homology $\mathbb{Z}$, with trivial $\mathbb{Z}\left[t, t^{-1}\right]$-action. This gives the universally twisted Floer homology

$$
\widehat{\widehat{H F}}\left(S^{1} \times S^{2} ; \mathbb{Z}\left[t, t^{-1}\right]\right) \cong \mathbb{Z},
$$

which is supported only in the torsion $\operatorname{Spin}^{c}$ structure $\mathfrak{s}_{0}$.

Now let's turn to an $\omega$-twisted example. We can view $S^{1} \times S^{2}$ as 0 -surgery on the unknot in $S^{3}$. Put $\mu$ a meridian for the unknot. Then $\mu$ defines a curve, also denoted $\mu$, in $S^{1} \times S^{2}$. Put $[\omega]=d \cdot \operatorname{PD}[\mu]$ for an integer $d$. The complex $\underline{\widehat{C F}}\left(S^{1} \times S^{2} ; \Lambda_{\omega}\right)$ is:

$$
0 \longrightarrow \Lambda \stackrel{t^{c}\left(1-t^{d}\right)}{\longrightarrow} \Lambda \longrightarrow 0
$$

for some $c \in \mathbb{R}$. Notice when $d \neq 0,\left(1-t^{d}\right)$ is invertible in $\Lambda$. Hence

$$
\underline{\widehat{H F}}\left(S^{1} \times S^{2} ; \Lambda_{\omega}\right)= \begin{cases}0 & \text { when } d \neq 0, \\ \Lambda \oplus \Lambda & \text { when } d=0 .\end{cases}
$$

As a final example, we prove a proposition regarding embedded 2-spheres in 3manifolds and $\omega$-twisted coefficients.

Lemma 2.1 Let $Y_{1}$ and $Y_{2}$ be a pair of closed oriented 3-manifolds and fix cohomology classes $\left[\omega_{i}\right] \in H^{2}\left(Y_{i} ; \mathbb{Z}\right)$. By the Mayer-Vietoris sequence we get a corresponding cohomology class $\omega_{1} \# \omega_{2} \in H^{2}\left(Y_{1} \# Y_{2} ; \mathbb{Z}\right) \cong H^{2}\left(Y_{1} ; \mathbb{Z}\right) \oplus H^{2}\left(Y_{2} ; \mathbb{Z}\right)$. Then we have an isomorphism of $\Lambda$-modules:

$$
\underline{\widehat{H F}}\left(Y_{1} \# Y_{2} ; \Lambda_{\omega_{1} \# \omega_{2}}\right) \cong \underline{\widehat{H F}}\left(Y_{1} ; \Lambda_{\omega_{1}}\right) \otimes_{\Lambda} \underline{\widehat{H F}}\left(Y_{2} ; \Lambda_{\omega_{2}}\right)
$$

Proof This follows readily from the methods of proof of Ozsváth-Szabó [16, Proposition 6.1] and the fact that $\Lambda$ is a field.

This allows us to prove:

Proposition 2.2 Let $S$ be an embedded nonseparating 2-sphere in a 3-manifold $Y$. Suppose that $[\omega] \in H^{2}(Y ; \mathbb{Z})$ is a cohomology class such that $\omega([S]) \neq 0$. Then $\underline{H F}^{+}\left(Y ; \Lambda_{\omega}\right)=0$. 
Proof Just as in the untwisted theory, $\underline{H F^{+}}(Y ; M)$ vanishes if and only if $\underline{\widehat{H F}}(Y ; M)$ vanishes, so it suffices to show that $\underline{\widehat{H F}}\left(Y ; \Lambda_{\omega}\right)=0$. Notice that $Y$ contains an $S^{1} \times S^{2}$ summand in its prime decomposition. Hence $Y \cong S^{1} \times S^{2} \# Y^{\prime}$ for some 3 -manifold $Y^{\prime}$. Now $\omega \in H^{2}(Y ; \mathbb{Z}) \cong H^{2}\left(S^{1} \times S^{2} ; \mathbb{Z}\right) \oplus H^{2}\left(Y^{\prime} ; \mathbb{Z}\right)$ corresponds to classes $\omega_{1} \in H^{2}\left(S^{1} \times S^{2} ; \mathbb{Z}\right)$ and $\omega_{2} \in H^{2}\left(Y^{\prime} ; \mathbb{Z}\right)$ with $\omega_{1}([S]) \neq 0$. We already know that $\underline{\widehat{H F}}\left(S^{1} \times S^{2} ; \Lambda_{\omega_{1}}\right)=0$ from the above calculation, so the proposition follows from Lemma 2.1 .

\section{Exact sequence for $\omega$-twisted Floer homology}

In this section we first prove a long exact sequence for the $\omega$-twisted Heegaard Floer homologies and then use it to prove Theorem 1.3. It is interesting to notice that there is a similar exact sequence in Monopole Floer homology with local coefficients; see Kronheimer, Mrowka, Ozsváth and Szabó [9, Section 5]. Our proof is a slight modification of the proof of the usual surgery exact sequence in Heegaard Floer homology. A good exposition of the original proof may be found in Ozsváth-Szabó [21].

Let $K \subset Y$ be framed knot in a 3-manifold $Y$ with framing $\lambda$ and meridian $\mu$. Given an integer $r$, let $Y_{r}(K)$ denote the 3-manifold obtained from $Y$ by performing Dehn surgery along the knot $K$ with framing $\lambda+r \mu$. Let $v(K)$ denote a small tubular neighborhood of the knot $K$ and $\eta \subset Y-v(K)$ be a closed curve in the knot complement. Then for any integer $r, \eta \subset Y-v(K) \subset Y_{r}(K)$ is a closed curve in the surgered manifold $Y_{r}(K)$, we denote its Poincaré dual by $\left[\omega_{r}\right] \in H^{2}\left(Y_{r}(K) ; \mathbb{Z}\right)$. Put $I=[0,1]$. Note that $\eta \times I$ represents a relative homology class in the cobordisms $W_{0}: Y \rightarrow Y_{0}(K), W_{1}: Y_{0}(K) \rightarrow Y_{1}(K)$ and $W_{2}: Y_{1}(K) \rightarrow Y$. So as in Section 2.1 it gives rise to homomorphisms between $\omega$-twisted Floer homologies

$$
\begin{gathered}
\underline{F}_{W_{0} ; \mathrm{PD}(\eta \times I)}^{+}: \underline{H F}^{+}\left(Y ; \Lambda_{\omega}\right) \rightarrow \underline{H F}^{+}\left(Y_{0}(K) ; \Lambda_{\omega_{0}}\right), \\
\underline{F}_{W_{1} ; \mathrm{PD}(\eta \times I)}^{+}: \underline{H F}{ }^{+}\left(Y_{0}(K) ; \Lambda_{\omega_{0}}\right) \rightarrow \underline{H F}^{+}\left(Y_{1}(K) ; \Lambda_{\omega_{1}}\right)
\end{gathered}
$$

and

$$
\underline{F}_{W_{2} ; \mathrm{PD}(\eta \times I)}^{+}: \underline{H F}^{+}\left(Y_{1}(K) ; \Lambda_{\omega_{1}}\right) \rightarrow \underline{H F}^{+}\left(Y ; \Lambda_{\omega}\right),
$$

where $\omega=\operatorname{PD}(\eta) \in H^{2}(Y ; \mathbb{Z})$. We denote the corresponding maps on the chain level by

$$
\underline{f}_{W_{0} ; \mathrm{PD}(\eta \times I)}^{+}, \quad \underline{f}_{W_{1} ; \mathrm{PD}(\eta \times I)}^{+} \quad \text { and } \quad \underline{f}_{W_{2} ; \mathrm{PD}(\eta \times I)}^{+}
$$

respectively. 
Theorem 3.1 The maps above form an exact sequence of $\Lambda$-modules:

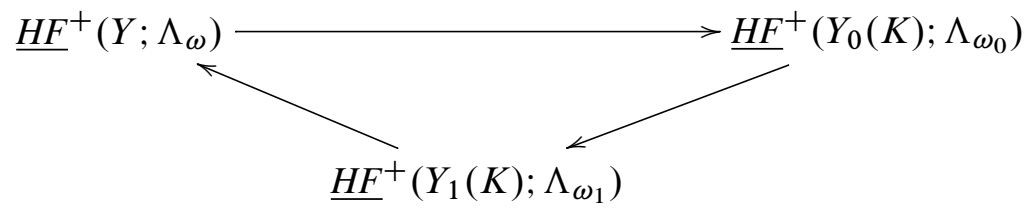

Furthermore, analogous exact sequences hold for "hat" versions as well.

Proof Find a Heegaard diagram $\left(\Sigma_{g},\left\{\alpha_{1}, \ldots, \alpha_{g}\right\},\left\{\beta_{1}, \ldots, \beta_{g}\right\}, z\right)$ compatible with the knot $K$. More precisely, $K$ lies in the handlebody specified by the $\beta$ curves and $\beta_{1}$ is a meridian for $K$. For each $i \geq 2$ let $\gamma_{i}, \delta_{i}$ be exact Hamiltonian isotopies of $\beta_{i}$. Let $\gamma_{1}=\lambda, \delta_{1}=\lambda+\mu$ be the 0 -framed and 1 -framed longitude of the knot $K$, respectively. We assume the Heegaard quadruple $(\Sigma, \boldsymbol{\alpha}, \boldsymbol{\beta}, \boldsymbol{\gamma}, \boldsymbol{\delta}, z)$ is weakly admissible in the sense of Ozsváth-Szabó [17]. It is easy to see that $Y_{\alpha \beta}=Y$, $Y_{\alpha \gamma}=Y_{0}(K), Y_{\alpha \delta}=Y_{1}(K)$, and $Y_{\beta \gamma} \cong Y_{\gamma \delta} \cong Y_{\beta \delta} \cong \#^{g-1} S^{2} \times S^{1}$.

Following Ozsváth-Szabó [19], we define a map

$$
h_{1}: \underline{C F}^{+}\left(Y ; \Lambda_{\omega}\right) \rightarrow \underline{C F}^{+}\left(Y_{1}(K) ; \Lambda_{\omega_{1}}\right)
$$

by counting holomorphic rectangles:

$$
h_{1}([\mathbf{x}, i])=\sum_{\mathbf{w} \in \mathbb{T}_{\alpha} \cap \mathbb{T}_{\delta}} \sum_{\substack{\varphi \in \pi_{2}\left(\mathbf{x}, \Theta_{\beta \gamma}, \Theta_{\gamma \delta}, \mathbf{w}\right) \\ \mu(\varphi)=0}} \# \mathcal{M}(\varphi)\left[\mathbf{w}, i-n_{z}(\varphi)\right] t^{\int[\varphi] \operatorname{PD}(\eta \times I)}
$$

where here $\mathcal{M}(\varphi)$ denotes the moduli space of (maps of) pseudo-holomorphic rectangles into $\operatorname{Sym}^{g}(\Sigma)$ allowing the conformal structure on the domain to vary. The notation $\int_{[\varphi]} \mathrm{PD}(\eta \times I)$ in the above formula requires some explanation. The Heegaard quadruple $(\Sigma, \boldsymbol{\alpha}, \boldsymbol{\beta}, \boldsymbol{\gamma}, \boldsymbol{\delta}, z)$ gives rise to a 4 -manifold $X_{\alpha \beta \gamma \delta}$ (as defined in Ozsváth-Szabó [20]) which can be thought of as the complement of two 1-complexes in the composite cobordism $Y \rightarrow Y_{0} \rightarrow Y_{1}$ and therefore we can consider $\operatorname{PD}(\eta \times I)$ as a class in $H^{2}\left(X_{\alpha \beta \gamma \delta}\right)$. Similar to the definition of the cobordism maps, the Whitney rectangle $\varphi$ determines a two-chain in $X_{\alpha \beta \gamma \delta}$ on which we may evaluate the 2 -form $\operatorname{PD}(\eta \times I)$, denoted $\int_{[\varphi]} \mathrm{PD}(\eta \times I)$. Similarly we define $h_{2}: \underline{C F^{+}}\left(Y_{0}(K) ; \Lambda_{\omega_{0}}\right) \rightarrow \underline{C F^{+}}\left(Y ; \Lambda_{\omega}\right)$ and $h_{3}: \underline{C F}{ }^{+}\left(Y_{1}(K) ; \Lambda_{\omega_{1}}\right) \rightarrow \underline{C F^{+}}\left(Y_{0}(K) ; \Lambda_{\omega_{0}}\right)$.

We claim that $h_{1}$ is a nullhomotopy of

$$
\underline{f}_{W_{1} ; \mathrm{PD}(\eta \times I)}^{+} \circ \underline{f}_{W_{0} ; \mathrm{PD}(\eta \times I)}^{+} .
$$


To see this, we consider the moduli space of holomorphic rectangles of Maslov index 1 . This moduli space can have six kinds of ends:

(1) Splicing holomorphic discs at one of its 4 corners.

(2) Splicing two holomorphic triangles. Triangles may be spliced in two ways: one triangle for $X_{\alpha \beta \gamma}$ and one triangle for $X_{\alpha \gamma \delta}$, or one triangle for $X_{\alpha \beta \delta}$ and one triangle for $X_{\beta \gamma \delta}$

Notice $\operatorname{PD}(\eta \times I)$ is 0 when restricted to the corners $Y_{\beta \gamma}$ and $Y_{\gamma \delta}$ : in fact, we can make $\eta \times I$ disjoint from these manifolds since $\eta$ may be pushed completely into the $\alpha$-handlebody, $U_{\alpha}$, by cellular approximation (see Figure 1).


Figure 1: Schematics of the 4-manifold $X_{\alpha \beta \gamma \delta}$ and its decompositions

This implies that

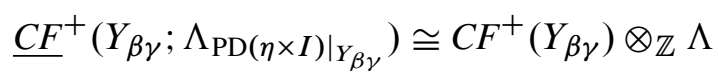

and all differentials are trivial (informally, we are using an "untwisted" count). For the end coming from splicing two holomorphic triangles, one for $X_{\alpha \beta \delta}$ and one for 
$X_{\beta \gamma \delta}$, it is also true that $\operatorname{PD}(\eta \times I)$ is 0 when restricted to the 4-manifold $X_{\beta \gamma \delta}$ (again, since $\eta$ may be pushed completely into $U_{\alpha}$ ). Therefore we are counting holomorphic triangles in $X_{\beta \gamma \delta}$ "without twisting". In Ozsváth-Szabó [16] it is shown that the untwisted counting of holomorphic triangles in $X_{\beta \gamma \delta}$ is zero. This leaves three terms remaining.

(1) Splicing a disc at corner $Y_{\alpha \beta}$ counted with twisting by $\left.\operatorname{PD}(\eta \times I)\right|_{Y_{\alpha \beta}}=[\omega]$, which corresponds to $h_{1} \circ \partial$.

(2) Splicing a disc at corner $Y_{\alpha \delta}$ counted with twisting by $\left.\operatorname{PD}(\eta \times I)\right|_{Y_{\alpha \delta}}=\left[\omega_{1}\right]$, which corresponds to $\partial \circ h_{1}$.

(3) Splicing two holomorphic triangles from $X_{\alpha \beta \gamma}$ and $X_{\alpha \gamma \delta}$ counted with twisting by $\mathrm{PD}(\eta \times I)$, which corresponds to $\underline{f}_{W_{1} ; \mathrm{PD}(\eta \times I)}^{+} \underline{f}_{W_{0} ; \mathrm{PD}(\eta \times I)}^{+}$.

From the fact that the moduli space must have total end zero, it is clear that the sum of the above 3 terms are zero, ie $h_{1}$ is a homotopy connecting

$$
\underline{f}_{W_{1} ; \mathrm{PD}(\eta \times I)}^{+} \circ \underline{f}_{W_{0} ; \mathrm{PD}(\eta \times I)}^{+}
$$

to the zero map. This shows that

$$
\underline{F}_{W_{1} ; \mathrm{PD}(\eta \times I)}^{+} \circ \underline{F}_{W_{0} ; \mathrm{PD}(\eta \times I)}^{+}=0
$$

on the homology level. The same argument shows that

$$
\underline{F}_{W_{2} ; \mathrm{PD}(\eta \times I)}^{+} \circ \underline{F}_{W_{1} ; \mathrm{PD}(\eta \times I)}^{+}=0 \quad \text { and } \quad \underline{F}_{W_{0} ; \mathrm{PD}(\eta \times I)}^{+} \circ \underline{F}_{W_{2} ; \mathrm{PD}(\eta \times I)}^{+}=0 .
$$

At last we prove that Equation (1) is exact. Using a homological algebra argument as in Ozsváth-Szabó [19] we need to show that $h \circ \underline{f}^{+}+\underline{f}^{+} \circ h$ is homotopic to the identity map. This can be done by counting holomorphic pentagons and noticing that we have a class $\mathrm{PD}(\eta \times I) \in H^{2}\left(X_{\alpha \beta \gamma \delta \beta^{\prime}}\right)$ similar to before (here $X_{\alpha \beta \gamma \delta \beta^{\prime}}$ is the complement of three 1-complexes in the composite cobordism $Y \rightarrow Y_{0} \rightarrow Y_{1} \rightarrow Y$ ) and that $\mathrm{PD}(\eta \times I)$ is zero when restricted to $Y_{\beta \gamma}, Y_{\gamma \delta}, Y_{\delta \beta^{\prime}}, X_{\beta \gamma \delta}, X_{\gamma \delta \beta^{\prime}}$ and $X_{\beta \gamma \delta \beta^{\prime}}$, similar to before. This shows that the counts there are "untwisted". From this observation one can easily see that everything in the proof of exactness in [19] can go through to our twisted version.

In the above theorem, the cohomology classes $\left[\omega_{r}\right]$ are integral. In practice one may need to use real cohomology class as well. In that situation, a given cohomology class $[\omega] \in H^{2}(Y ; \mathbb{R})$ can be expressed as a finite sum

$$
[\omega]=\sum a_{i} \mathrm{PD}\left(\eta_{i}\right)
$$


where the $\eta_{i}$ are closed curves in the knot complement and $a_{i} \in \mathbb{R}$. Each $\eta_{i}$ can be viewed as a closed curve in $Y_{r}(K)$, so the expression $\sum a_{i} \mathrm{PD}\left(\eta_{i}\right)$ also gives a real cohomology class in $Y_{r}(K)$, denote by $\left[\omega_{r}\right] \in H^{2}\left(Y_{r}(K) ; \mathbb{R}\right)$. In the cobordism $W_{r}$,

$$
\sum a_{i} \mathrm{PD}\left(\eta_{i} \times I\right)
$$

is a real cohomology class in $H^{2}\left(W_{r} ; \mathbb{R}\right)$, hence gives rise to homomorphism between $\omega$-twisted Floer homologies. With this understood, it is easy to see that an analogue Theorem 3.1 still holds.

Remark 3.2 The exact sequence in Theorem 3.1 depends on $\eta$, not just its Poincaré dual $[\omega] \in H^{2}\left(Y ; \mathbb{Z}\right.$ ). In fact if we take another closed curve $\eta^{\prime}=\eta+k \cdot \mu$ (where $\mu$ is a meridian of $K)$, this doesn't change $[\omega]$, but may change $\left[\omega_{0}\right],\left[\omega_{1}\right]$ and the exact sequence. For example, take $K \subset S^{3}$ to be the unknot and $\eta=k \cdot \mu$ in the knot complement, then $\left[\omega_{0}\right]$ is $k$ times the generator of $H^{2}\left(S^{2} \times S^{1} ; \mathbb{Z}\right)$. When $k \neq 0$, the corresponding exact sequence for the hat version is:

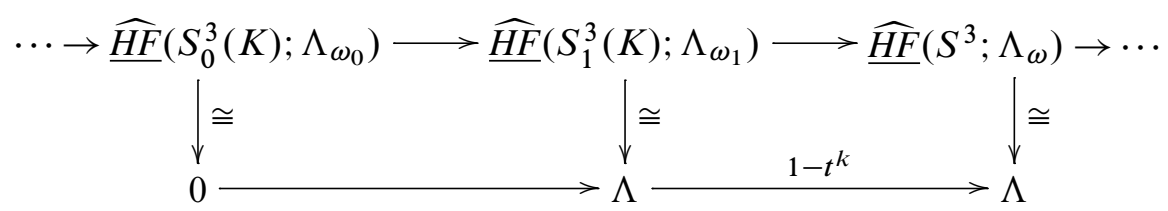

Clearly it depends on $k$. When $k=0$, the exact sequence is obtained from the corresponding exact sequence for untwisted Heegaard floer homology by tensoring with $\Lambda$.

Ozsváth and Szabó [14] used another version of twisted Floer homology $\underline{H F}^{+}(Y ;[\omega])$, which is defined by using the $\mathbb{Z}\left[H^{1}(Y ; \mathbb{Z})\right]$-module $\mathbb{Z}[\mathbb{R}]$. The $\omega$-twisted Floer homology we used in this paper can be viewed as a completion of $\operatorname{HF}^{+}(Y ;[\omega])$. It is easy to see that there is a similar exact sequence in their context. More precisely, we have the following exact sequence:

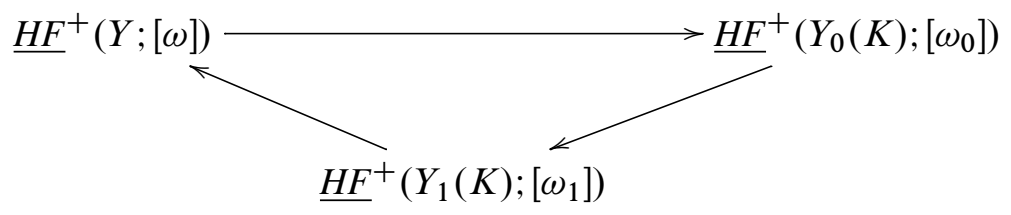

With the above exact sequences in place, we can now prove Theorem 1.3. We merely mimic Ozsváth and Szabó's proof of [18, Theorem 5.2].

Proof of Theorem 1.3 For a given cohomology class $[\omega] \in H^{2}(Y ; \mathbb{Z})$ with $\omega(F)=$ $d \neq 0$, choose a closed curve $\eta \subset Y$ such that its Poincaré dual $\operatorname{PD}(\eta)$ equals $[\omega]$. 
Since the mapping class group of a torus is generated as a monoid by right-handed Dehn twists along nonseparating curves (see Humphries [6] or Ozsváth-Szabó [18, Theorem 2.2]), we can connect $Y$ to the three-manifold $S_{0}^{3}(T)$, which is obtained from $S^{3}$ by performing 0 -surgery on the right-handed trefoil, by a sequence of torus bundles

$$
\pi_{i}: Y^{i} \rightarrow S^{1}
$$

and cobordisms

$$
Y=Y^{0} \stackrel{W_{0}}{\longrightarrow} Y^{1} \stackrel{W_{1}}{\longrightarrow} \cdots \stackrel{W_{n-1}}{\longrightarrow} Y^{n}=S_{0}^{3}(T)
$$

such that the monodromy of $Y^{i+1}$ differs from that of $Y^{i}$ by a single right-handed Dehn twist along a nonseparating knot $K_{i}$ which lies in a fiber $F_{i}$ of $\pi_{i}$. The curve $\eta \subset Y$ induces curves $\eta_{i} \subset Y^{i}$ which can be assumed disjoint from $K_{i}$. In this way, we get a sequence of cohomology classes $\omega_{i}=\operatorname{PD}\left(\eta_{i}\right) \in H^{2}\left(Y^{i} ; \mathbb{Z}\right)$ such that $\omega_{i}\left(F_{i}\right)=d \neq 0$. The cobordism $W_{i}$ is obtained by attaching a single 2-handle to $Y^{i} \times I$ along the knot $K_{i}$ with framing -1 (with respect to the framing $K_{i}$ inherits from the fiber $F_{i}$ ). Since $\eta_{i}$ is disjoint from $K_{i}, \eta_{i} \times I$ defines a relative homology class $\left[\eta_{i} \times I\right] \in H_{2}\left(W_{i}, \partial W_{i} ; \mathbb{Z}\right)$ and hence its Poincaré dual gives rise to homomorphisms between $\omega$-twisted Floer homologies:

$$
\underline{F}_{W_{i} ; \mathrm{PD}\left(\eta_{i} \times I\right)}^{+}: \underline{H F}^{+}\left(Y^{i} ; \Lambda_{\omega_{i}}\right) \rightarrow \underline{H F}^{+}\left(Y^{i+1} ; \Lambda_{\omega_{i+1}}\right)
$$

We claim that these maps are all isomorphisms. Notice that $Y^{i+1}=\left(Y^{i}\right)_{-1}\left(K_{i}\right)$ where the 0 -framing of $K_{i}$ is defined to be the framing $K_{i}$ inherits from the fiber, $F_{i}$. Now consider $\left(Y^{i}\right)_{0}\left(K_{i}\right)$. This manifold contains a $2-$ sphere $S_{i}$ (which is obtained from $F_{i}$ by surgering along $\left.K_{i}\right)$ and also an induced curve $\eta_{i}$ such that $\eta_{i} \cdot S_{i}=d \neq 0$, therefore $\underline{H F}^{+}\left(\left(Y^{i}\right)_{0}\left(K_{i}\right) ; \Lambda_{\mathrm{PD}\left(\eta_{i}\right)}\right)=0$ by Proposition 2.2. Equation (1) now proves the claim.

This shows that

$$
\underline{H F}^{+}\left(Y ; \Lambda_{\omega}\right) \cong \underline{H F}^{+}\left(S_{0}^{3}(T) ; \Lambda_{\mathrm{PD}(\eta)}\right)
$$

where $\eta$ is the induced curve in $S_{0}^{3}(T)$. We now identify the latter group. For simplicity we write $\omega=\operatorname{PD}(\eta)$. Identifying $\mathbb{Q}\left[H^{1}\left(S_{0}^{3}(T) ; \mathbb{Z}\right)\right]$ with $\mathbb{Q}\left[t, t^{-1}\right]$, Ozsváth and Szabó show in [13] that there is an identification of $\mathbb{Q}\left[t, t^{-1}\right]$-modules:

$$
\underline{H F}_{k}^{+}\left(S_{0}^{3}(T) ; \mathbb{Q}\left[t, t^{-1}\right]\right) \cong \begin{cases}\mathbb{Q} & \text { if } k \equiv-1 / 2(\bmod 2) \text { and } k \geq-1 / 2 \\ \mathbb{Q}\left[t, t^{-1}\right] & \text { if } k=-3 / 2 \\ 0 & \text { otherwise }\end{cases}
$$


Where the left hand group is the universally twisted Heegaard Floer homology of $S_{0}^{3}(T)$, the $\mathbb{Q}$ 's on the right are trivial $\mathbb{Q}\left[H^{1}\left(S_{0}^{3}(T) ; \mathbb{Z}\right)\right]$-modules, and $\mathbb{Q}\left[t, t^{-1}\right]$ is a module over itself in the natural way. By definition,

$$
\underline{C F}^{+}\left(S_{0}^{3}(T) ; \Lambda_{\omega}\right)=\underline{C F}^{+}\left(S_{0}^{3}(T) ; \mathbb{Q}\left[t, t^{-1}\right]\right) \otimes_{\mathbb{Q}\left[t, t^{-1}\right]} \Lambda_{\omega} .
$$

Notice $\mathbb{Q}\left[t, t^{-1}\right]$ is a principal ideal domain, so by the universal coefficient theorem (see for instance of Hilton and Stammbach [5, Theorem 2.5]) there is an exact sequence:

$$
\begin{aligned}
0 \rightarrow \underline{H F}^{+}\left(S_{0}^{3}(T) ; \mathbb{Q}\left[t, t^{-1}\right]\right) \otimes_{\mathbb{Q}\left[t, t^{-1}\right]} \Lambda_{\omega} \\
\rightarrow \underline{H F^{+}}\left(S_{0}^{3}(T) ; \Lambda_{\omega}\right) \rightarrow \operatorname{Tor}_{1}^{\mathbb{Q}\left[t, t^{-1}\right]} \underline{H F}^{+}\left(S_{0}^{3}(T), \Lambda\right) \rightarrow 0
\end{aligned}
$$

We need only compute $\operatorname{Tor}_{q}^{\mathbb{Q}\left[t, t^{-1}\right]}\left(\mathbb{Z}, \Lambda_{\omega}\right)$ for $q=0,1$. Start with the free $\mathbb{Q}\left[t, t^{-1}\right]-$ resolution of $\mathbb{Z}$ :

$$
0 \longrightarrow \mathbb{Q}\left[t, t^{-1}\right] \stackrel{1-t}{\longrightarrow} \mathbb{Q}\left[t, t^{-1}\right] \longrightarrow \mathbb{Z} \longrightarrow 0
$$

Tensoring this complex over $\mathbb{Q}\left[t, t^{-1}\right]$ with $\Lambda_{\omega}$ and augmenting gives the complex:

$$
0 \longrightarrow \Lambda \stackrel{1-t^{d}}{\longrightarrow} \Lambda \longrightarrow 0
$$

Since we're working over $\Lambda$ and $d \neq 0$, the middle map is an isomorphism and we see that

$$
\operatorname{Tor}_{q}^{\mathbb{Z}\left[t, t^{-1}\right]}\left(\mathbb{Z}, \Lambda_{\omega}\right)=0
$$

for all $q$. From the above exact sequence, we obtain an isomorphism of $\Lambda$-modules $\underline{H F}^{+}\left(S_{0}^{3}(T) ; \Lambda_{\omega}\right) \cong \Lambda$. Therefore

$$
\underline{H F}^{+}\left(Y ; \Lambda_{\omega}\right) \cong \Lambda .
$$

It is worth noting that alternate proofs of this theorem and Proposition 2.2 are possible through the use of inadmissible diagrams, which have been explored by $\mathrm{Wu}$ in [23] as well as by Lekili in [10].

\section{References}

[1] Y Ai, Y Ni, Two applications of twisted Floer homology, Int. Math. Res. Not. (2009) 3726-3746 MR2539188

[2] J A Baldwin, Heegaard Floer homology and genus one, one-boundary component open books, J. Topol. 1 (2008) 963-992 MR2461862 
[3] H Cartan, S Eilenberg, Homological algebra, Princeton Landmarks in Math., Princeton Univ. Press (1999) MR1731415 With an appendix by D A Buchsbaum, Reprint of the 1956 original

[4] P Ghiggini, Knot Floer homology detects genus-one fibred knots, Amer. J. Math. 130 (2008) 1151-1169 MR2450204

[5] P J Hilton, U Stammbach, A course in homological algebra, Graduate Texts in Math. 4, Springer, New York (1971) MR0346025

[6] S P Humphries, Generators for the mapping class group, from: "Topology of lowdimensional manifolds (Proc. Second Sussex Conf., Chelwood Gate, 1977)”, (R A Fenn, editor), Lecture Notes in Math. 722, Springer, Berlin (1979) 44-47 MR547453

[7] S Jabuka, T E Mark, Product formulae for Ozsváth-Szabó 4-manifold invariants, Geom. Topol. 12 (2008) 1557-1651 MR2421135

[8] P Kronheimer, T Mrowka, Monopoles and three-manifolds, New Math. Monogr. 10, Cambridge Univ. Press (2007) MR2388043

[9] P Kronheimer, T Mrowka, P Ozsváth, Z Szabó, Monopoles and lens space surgeries, Ann. of Math. (2) 165 (2007) 457-546 MR2299739

[10] Y Lekili, Heegaard Floer homology of broken fibrations over the circle arXiv: 0903.1773

[11] Y Ni, Knot Floer homology detects fibred knots, Invent. Math. 170 (2007) 577-608 MR2357503

[12] Y Ni, Heegaard Floer homology and fibred 3-manifolds, Amer. J. Math. 131 (2009) 1047-1063 MR2543922

[13] P Ozsváth, Z Szabó, Absolutely graded Floer homologies and intersection forms for four-manifolds with boundary, Adv. Math. 173 (2003) 179-261 MR1957829

[14] P Ozsváth, Z Szabó, Holomorphic disks and genus bounds, Geom. Topol. 8 (2004) 311-334 MR2023281

[15] P Ozsváth, Z Szabó, Holomorphic disks and knot invariants, Adv. Math. 186 (2004) 58-116 MR2065507

[16] P Ozsváth, Z Szabó, Holomorphic disks and three-manifold invariants: properties and applications, Ann. of Math. (2) 159 (2004) 1159-1245 MR2113020

[17] P Ozsváth, Z Szabó, Holomorphic disks and topological invariants for closed threemanifolds, Ann. of Math. (2) 159 (2004) 1027-1158 MR2113019

[18] P Ozsváth, Z Szabó, Holomorphic triangle invariants and the topology of symplectic four-manifolds, Duke Math. J. 121 (2004) 1-34 MR2031164

[19] P Ozsváth, Z Szabó, On the Heegaard Floer homology of branched double-covers, Adv. Math. 194 (2005) 1-33 MR2141852 
[20] P Ozsváth, Z Szabó, Holomorphic triangles and invariants for smooth four-manifolds, Adv. Math. 202 (2006) 326-400 MR2222356

[21] P Ozsváth, Z Szabó, Lectures on Heegaard Floer homology, from: "Floer homology, gauge theory, and low-dimensional topology", (D A Ellwood, P Ozsváth, A Stipsicz, Z Szabó, editors), Clay Math. Proc. 5, Amer. Math. Soc. (2006) 29-70 MR2249248

[22] J Rasmussen, Floer homology and knot complements, $\mathrm{PhD}$ thesis, Harvard University (2003) arXiv:math.GT/0306378

[23] Z Wu, Perturbed Floer homology of some fibered three-manifolds, Algebr. Geom. Topol. 9 (2009) 337-350 MR2482081

Department of Mathematics, Tsinghua University Beijing 100084, China

Department of Mathematics, Columbia University MC 4406, 2990 Broadway, New York, NY 10027, United States yhai@math.tsinghua.edu.cn, tpeters@math.columbia.edu http://www.math.columbia.edu/ tpeters/

Received: 22 November $2008 \quad$ Revised: 28 October 2009 